\title{
A REMARK ON INHERENT DIFFERENTIABILITY
}

\author{
MICHAEL H. FREEDMAN AND ZHENG-XU HE ${ }^{1}$
}

(Communicated by Haynes R. Miller)

\begin{abstract}
Harrison's analysis of $C^{r}$-diffeomorphisms which are not conjugate to $C^{s}$-diffeomorphisms for $s>r>0$ is extended to dimension $=4$. Also topological conjugacy may be generalized to an arbitrary change of differentiable structure. Combining these statements yields: for any smooth manifold of dimension $\geq 2$ there is a $C^{r}$-diffeomorphism which is not a $C^{s}$ diffeomorphism w.r.t. any smooth structure.
\end{abstract}

Our seminar had the pleasure of reading Jenny Harrison's papers [H1 and H2] in which she constructs examples of $C^{r}$-diffeomorphisms $f_{n, r}$ of $D^{n} \times S^{1}$ which are not topologically conjugate to $C^{s}$-diffeomorphisms for all $s>r>0$ and $n+1 \neq 1$ or 4. It is a result of Denjoy [D] that $C^{2}$-diffeomorphisms of $S^{1}$ are conjugate into $C^{\infty}$; however, the cases where $n+1=4$ are not actually exceptional. A baby version of Rob Kirby's famous "torus trick" may be inserted into Harrison's proof to replace the difficult fact that when $n+1 \neq 4$ any homeomorphisms of $D^{n} \times S^{1}$ may be approximated by $C^{\infty}$-diffeomorphisms. ${ }^{2}$ Modified in this way, the argument is elementary and self-contained and applies equally well in dimension $=4$.

In briefest outline, Harrison shows that when the rotation numbers along the orbits of $f_{n, r}$ vary as rapidly as $C^{r}$ permits, volume considerations prevent "spreading out" the orbits by a topological conjugation so that they might vary in a more gradual $C^{s}$ fashion. The technical device used to link the dynamics with volume requires the conjugating homeomorphism to be near the identity. Actually, the proof only uses nearness in the $S^{1}$ factor and this may be obtained by lifting everything to a finite cover.

The conjugating homeomorphism $h$ may be assumed, after composing with a diffeomorphism if necessary, to be homotopic to the identity. Fix a lift $f_{n, r, l}$ of $f_{n, r}$ along the $l$-fold covering $D^{n} \times S^{1} \stackrel{\pi_{l}}{\rightarrow} D^{n} \times S^{1}$ and use the homotopy to define lifts $h_{l}$ of $h$ also to the $l$-fold cover. If $h \circ f_{n, r} \circ h^{-1}$ is $C^{s}$ so is any lift $\left(h \circ f_{n, r} \circ h^{-1}\right)_{l}=h_{l} \circ f_{n, r, l} \circ h_{l}^{-1}$. Thus $h_{l}$ conjugates the $C^{r}$-diffeomorphism $f_{n, r, l}$ into $C^{s}$. But Kirby's observation is that the distance between $h_{l}: D^{n} \times S^{1} \hookleftarrow$ and $\operatorname{id}_{D^{n} \times S^{1}}$ (as measured in the $S^{1}$-factor!) approaches zero as $l$ approaches infinity. For $l$ sufficiently large $h_{l}$ is closer to the identity (in the $S^{1}$-factor) than the constant $(=1 / 12)$ appearing in Harrison's proof. We observe that all the lifts $f_{n, r, l}$ satisfy the criteria of Harrison's construction so that the constant does not depend on the

Received by the editors June 1, 1987 and, in revised form February 12, 1988.

1980 Mathematics Subject Classification (1985 Revision). Primary 57D50, 58C99; Secondary 57D30, 58F99.

1 The authors were supported in part by NSF DMS86-1037; the MacArthur Foundation; and DARPA/ACMP No. 86-A227500.

${ }^{2} \mathrm{This}$ fact is false in dimension $=4$. 
choice of $l$. Thus $f_{n, r}$ cannot be conjugated into $C^{s}$ because then $f_{n, r, l}$ would be conjugate by $h_{l}$ into $C^{s}$, a possibility excluded by the closeness of $h_{l}$ to id (in the $S^{1}$-factor) when $l$ is large.

Further, Harrison's constructions on arbitrary manifolds also extend to dimension $=4$. To describe this it is convenient to back up and slightly reorganize her proof so that "distance" is measured merely by a smooth map-rather than smooth submersion - to the circle. In this way we will not arrive at difficult approximation questions. This simplified approach leads to the somewhat stronger conclusion (see Theorem 2.1) that Harrison's $C^{r}$-diffeomorphisms are not $C^{s}$, for any $s>r$, with respect to any differentiable structure on the underlying manifold. ${ }^{3}$ (Note that $h \circ f \circ h^{-1}$ is $C^{s}$ with respect to a differentiable structure $\Gamma$ if and only if $f$ was already in $C^{s}$ with respect to $h^{*}(\Gamma)$.)

1. Let $n$ be any integer $\geq 1$, and let $r$ be any real number $>0$. Let us recall Harrison's construction of $f_{n, r}: D^{n} \times S^{1} \rightarrow D^{n} \times S^{1}$. Take $\alpha_{k}$ to be a sequence of positive integers such that $\alpha_{k} \geq 2, \sum_{k=1}^{\infty}\left(1 / \alpha_{k}\right)^{n / r}<\infty$ (hence $\alpha_{k} \rightarrow \infty$ ), and

$$
\sum_{k=1}^{\infty}\left(\frac{1}{\alpha_{k}}\right)^{n / s}=\infty \quad \forall s>r .
$$

(We can take for example $\alpha_{k}=$ the integer part of $\left(\left(k \log ^{2} k\right) / c\right)^{r / n}+2$ for some constant $c>0$.) By [H2, Lemma 1] and its proof, there exists a sequence $a_{k}$ with $a_{k}>0,1 / \alpha_{k} a_{k}^{r} \rightarrow 0$, and

$$
\sum_{k} a_{k}^{n}<\infty
$$

By the Packing Lemma [H2, Lemma 2], there exist disjoint closed $n$-cubes $Q_{k}$ with edges of length $a_{k}$ in $\mathbf{R}^{n}$ whose centers converge to the origin. Dropping out a finite number of terms in the sequences $\alpha_{k}$ and $a_{k}$, we may assume that

$$
\bigcup_{k=1}^{\infty} Q_{k} \subseteq \stackrel{\circ}{D}^{n}\left(\frac{1}{2}\right) \equiv\left\{x \in \mathbf{R}^{n} ;|x|<\frac{1}{2}\right\} \text {. }
$$

Let $\Phi: \mathbf{R}^{n} \rightarrow[0,1]$ be a smooth $\left(C^{\infty}\right)$ bump function with support in $\stackrel{\circ}{D}^{n}(1 / 2)$ such that $\Phi(0)=1$. Then $f_{n, r}: D^{n} \times S^{1} \rightarrow D^{n} \times S^{1}$ is defined by

$$
f_{n, r}(x, t)= \begin{cases}\left(x, t+\left(1 / \alpha_{k}\right) \Phi\left(\left(x-q_{k}\right) / a_{k}\right)\right) & \text { if } x \in Q_{k} \\ (x, t) & \text { otherwise }\end{cases}
$$

where $x \in D^{n}, t \in S^{1}=\mathbf{R} / 2 \pi$, and $q_{k}$ is the center of the cube $Q_{k} . f_{n, r}$ is a $C^{r}$-diffeomorphism (see [H2, p. 253]) with

$$
f_{n, r}=\mathrm{id} \text { near } \partial Q_{k} \times S^{1}
$$

and

$$
f_{n, r}\left(q_{k}, t\right)=\left(q_{k}, t+1 / \alpha_{k}\right) \quad \forall t \in S^{1}
$$

\footnotetext{
${ }^{3}$ In particular note that when $n=3, D^{n} \times S^{1}$ may have differential structures which are not product structures. This means Fubini's theorem (as used in Harrison's argument) will not be available, necessitating the above mentioned reorganization.
} 
As we remarked in the introduction, a combination of Harrison's argument and the idea of Kirby's torus trick shows that $f_{n, r}$ can not be conjugated to any $C^{s}$ diffeomorphism for $s>r$. In the following section, we will give a general argument which shows particularly that $f_{n, r}$ is not $C^{s}$ with respect to any differential structure on $D^{n} \times S^{1}$.

2. Let $M^{n+1}$ be any $\left((n+1)\right.$-dimensional) $C^{\infty}$-manifold, $n+1 \geq 2$. Let $\Psi$ be a smooth embedding of $D^{n} \times S^{1}$ to $M^{n+1}$. Define $f: M^{n+1} \rightarrow M^{n+1}$ by

$$
f(p)= \begin{cases}\Psi \circ f_{n, r} \circ \Psi^{-1}(p) & \text { if } p \in \Psi\left(D^{n} \times S^{1}\right), \\ p & \text { otherwise. }\end{cases}
$$

Then $f$ is obviously a $C^{r}$-diffeomorphism.

THEOREM 2.1. For any $C^{\infty}$-manifold $N^{n+1}$ homeomorphic to $M^{n+1}$ and for any homeomorphism $h: M^{n+1} \rightarrow N^{n+1}$, the mapping $h \circ f \circ h^{-1}: N^{n+1} \rightarrow N^{n+1}$ is not $C^{s}$ for any real number $s>r$.

The proof is based on the following

LEMMA 2.2. Let $\eta: S^{1} \rightarrow S^{1}$ be a fixed point free homeomorphism with $\eta^{\alpha}=$ id for some integer $\alpha \geq 2$, and let $\lambda: S^{1} \rightarrow S^{1}=\mathrm{R} / 2 \pi Z$ be a homotopy equivalence. Suppose $\tilde{\eta}: \mathbf{R}^{1} \rightarrow \mathbf{R}^{1}$ and $\tilde{\lambda}: \mathbf{R}^{1} \rightarrow \mathbf{R}^{1}$ are some lifts of $\eta$ and $\lambda$ respectively. Then there exist a finite union $U$ of disjoint closed intervals contained in the interval $[0,2 \pi)$ such that: (i) the total length $|U|$ of $U$ is $>1$ and, (ii) for any $u \in U$, there is some $t(u) \in \tilde{\lambda}^{-1}(u)$ satisfying

$$
|\tilde{\lambda}(\tilde{\eta}(t(u)))-\tilde{\lambda}(t(u))| \geq 1 / \alpha .
$$

PROOF. First we remark that we can assume that $\lambda$ is homotopic to $\operatorname{id}_{S^{1}}$, and since $\eta$ is fixed point free, $\eta \simeq \operatorname{id}_{S^{1}}$. In this way, $\tilde{\eta}(t+2 \pi)=\tilde{\eta}(t)+2 \pi$, $\tilde{\lambda}(t+2 \pi)=\tilde{\lambda}(t)+2 \pi$. Since $\eta^{\alpha}=\mathrm{id}$ and $\eta$ is fixed point free, we have $\tilde{\eta}^{\alpha}(t)=t+2 \pi j$, for some integer $j \neq 0$. Let us start with the case $j>0$. The proof is patterned on [H1, pp. 88-90] where the map $\pi_{2} \circ f^{\prime \prime}$ corresponds to our $\lambda$. Define the "last point" function $l: \mathbf{R}^{1} \rightarrow \mathbf{R}^{1}$ by $l(u)=\max \left\{t ; t \in \tilde{\lambda}^{-1}(u)\right\}$ and let $L=l\left(\mathbf{R}^{\mathbf{1}}\right)$. Then $l$ is increasing, $\tilde{\lambda} \circ l=\mathrm{id}, l \circ \tilde{\lambda} \geq \mathrm{id}, l(u+2 \pi)=l(u)+2 \pi$, and $L$ is lower semiclosed in the sense that if $t_{i} \in L, t_{i} \searrow t_{\infty}$, then $t_{\infty} \in L$. Define the "next last point" function $m: \mathbf{R}^{1} \rightarrow \mathbf{R}^{1}$ by $m(t)=\min \{\tau \in L ; \tau \geq t\}$. Then $m$ is nondecreasing, $m\left(\mathbf{R}^{1}\right) \subseteq L$, $m(t) \geq t, m(t+2 \pi)=m(t)+2 \pi, m \circ l(u)=l(u)$, and $\tilde{\lambda}(m(t)) \leq \tilde{\lambda}(t)$. Now define $\tilde{\eta}^{\prime}: \mathbf{R}^{1} \rightarrow \mathbf{R}^{1}$ by $\tilde{\eta}^{\prime}=\tilde{\lambda} \circ m \circ \tilde{\eta} \circ l$. Then $\tilde{\eta}^{\prime}$ is nondecreasing, $\tilde{\eta}^{\prime}(\tilde{\lambda} \circ l(u)) \leq \tilde{\lambda}(\tilde{\eta} \circ l(u))$, and

$$
\begin{aligned}
\tilde{\eta}^{\prime \alpha}(u) & =(\tilde{\lambda} \circ m \circ \tilde{\eta} \circ l)^{\alpha}(u)=\tilde{\lambda} \circ(m \circ \tilde{\eta} \circ l \circ \tilde{\lambda})^{\alpha-1} \circ m \circ \tilde{\eta} \circ l(u) \\
& \geq \tilde{\lambda} \circ(m \circ \tilde{\eta})^{\alpha-1} \circ m \circ \tilde{\eta}(l(u))=\tilde{\lambda} \circ(m \circ \tilde{\eta})^{\alpha}(l(u)) \geq \tilde{\lambda} \circ m \circ \tilde{\eta}^{\alpha} \circ l(u) \\
& =\tilde{\lambda} \circ m(l(u)+2 \pi j)=\tilde{\lambda} \circ m \circ l(u)+2 \pi j=u+2 \pi j .
\end{aligned}
$$

Let $u_{0}=0, u_{1}=\tilde{\eta}^{\prime}\left(u_{0}\right), \ldots, u_{\alpha-1}=\tilde{\eta}^{\prime}\left(u_{\alpha-2}\right), u_{\alpha}=\tilde{\eta}^{\prime}\left(u_{\alpha-1}\right)$. Then $u_{\alpha}=$ $\tilde{\eta}^{\prime \alpha}(0) \geq 2 \pi j \geq 2 \pi$. Let $u_{i_{0}}, i_{0} \leq \alpha$, be the element such that $u_{i_{0}} \geq 2 \pi$ and $u_{i_{0}-1}<2 \pi$. Set $u_{0}^{\prime}=u_{0}, u_{1}^{\prime}=u_{1}, \ldots, u_{i_{0}-1}^{\prime}=u_{i_{0}-1}, u_{i_{0}}^{\prime}=2 \pi$. Let $U$ be the union of closed intervals $\left[u_{i}^{\prime}, u_{i}^{\prime}+\frac{1}{2}\left(u_{i+1}^{\prime}-u_{i}^{\prime}\right)\right]$ for $0 \leq i \leq i_{0}-1$ which satisfy $u_{i+1}^{\prime}-u_{i}^{\prime} \geq 2 \pi / 2 \alpha$. Since $i_{0} \leq \alpha,|U| \geq \frac{1}{2}(2 \pi / 2)>1$. 
For any $u \in\left[u_{i}^{\prime}, u_{i}^{\prime}+\frac{1}{2}\left(u_{i+1}^{\prime}-u_{i}^{\prime}\right)\right]$, let $t(u)=l(u)$, then

$$
\begin{aligned}
\tilde{\lambda}(\tilde{\eta}(t(u)))-\tilde{\lambda}(t(u)) & =\tilde{\lambda}(\tilde{\eta} \circ l(u))-\tilde{\lambda} \circ l(u) \\
& \geq \tilde{\eta}^{\prime}(\tilde{\lambda} \circ l(u))-u=\tilde{\eta}^{\prime}(u)-u \\
& \geq \tilde{\eta}^{\prime}\left(u_{i}^{\prime}\right)-\left(u_{i}^{\prime}+\frac{1}{2}\left(u_{i+1}^{\prime}-u_{i}^{\prime}\right)\right) \\
& \geq u_{i+1}^{\prime}-\left(u_{i}^{\prime}+\frac{1}{2}\left(u_{i+1}^{\prime}-u_{i}^{\prime}\right)\right) \\
& =\frac{1}{2}\left(u_{i+1}^{\prime}-u_{i}^{\prime}\right) \geq \frac{1}{2}(2 \pi / 2 \alpha)>1 / \alpha .
\end{aligned}
$$

This proves the lemma in the case $j>0$. When $j<0$, it is enough to replace $\eta, \lambda, \tilde{\eta}$ and $\tilde{\lambda}$ by $\beta \circ \eta \circ \beta, \beta \circ \lambda \circ \beta, \tilde{\beta} \circ \tilde{\eta} \circ \tilde{\beta}$ and $\tilde{\beta} \circ \tilde{\lambda} \circ \tilde{\beta}$ respectively, where $\beta(t)=-t$, $t \in S^{1}$ and $\tilde{\beta}(t)=-t, t \in \mathbf{R}^{1}$, and apply the result proved for the case $j>0$.

PROOF OF THEOREM 2.1. Suppose contrarily that there were some homeomorphism $h: M^{n+1} \rightarrow N^{n+1}$ such that $h \circ f \circ h^{-1}: N^{n+1} \rightarrow N^{n+1}$ is $C^{s}$ for some $s>r$. Let $h^{\prime}=h \circ \psi: D^{n} \times S^{1} \rightarrow N^{n+1}$, then $h^{\prime}$ is a topological embedding such that $h^{\prime} \circ f_{n, r} \circ h^{\prime-1}=h \circ f \circ h^{-1}$ is a $C^{s}$ map from int $h^{\prime}\left(D^{n} \times S^{\prime}\right)=h^{\prime}\left(\stackrel{\circ}{D}^{n} \times S^{1}\right) \subseteq N^{n+1}$ to itself, where $\stackrel{\circ}{D}^{n}=\operatorname{int} D^{n}$. We will show this yields a contradiction.

Let $\Lambda: h^{\prime}\left(\stackrel{\circ}{D}^{n} \times S^{1}\right) \rightarrow S^{1}=\mathbf{R} / 2 \pi Z$ be a smooth approximation of $\pi_{2} \circ h^{\prime-1}$, where $\pi_{2}: D^{n} \times S^{1} \rightarrow S^{1}$ is the projection onto the second factor. We will denote by $\tilde{X}$ the universal cover of $X$, for any manifold $X$. So $\tilde{X}$ is a $C^{\infty}$-manifold if $X$ is. Let

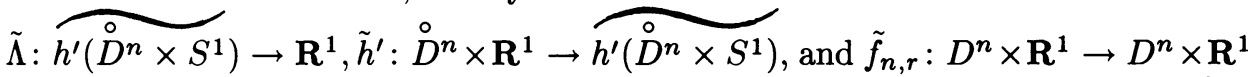
be some lifts of $\Lambda, h^{\prime}$ and $f_{n, r}$ respectively. By (1.4) and (1.5) we may choose $\tilde{f}_{n, r}$ to satisfy:

$$
\tilde{f}_{n, r}=\mathrm{id} \quad \text { near } \partial Q_{k} \times \mathbf{R}^{1} \quad \forall k \geq 1 .
$$

For any $k \geq 1$, let $\alpha=\alpha_{k}, \eta(t)=\eta_{k}(t)=\pi_{2} \circ f_{n, r}\left(q_{k}, t\right): S^{1} \rightarrow S^{1}, \tilde{\eta}(t)=$ $\tilde{\eta}_{k}(t)=\pi_{2} \circ \tilde{f}_{n, r}\left(q_{k}, t\right): \mathbf{R}^{1} \rightarrow \mathbf{R}^{1}, \lambda(t)=\lambda_{k}(t)=\Lambda \circ h^{\prime}\left(q_{k}, t\right): S^{1} \rightarrow S^{1}$, and $\tilde{\lambda}=\tilde{\lambda}_{k}(t)=\tilde{\Lambda} \circ \tilde{h}^{\prime}\left(q_{k}, t\right): \mathbf{R}^{1} \rightarrow \mathbf{R}^{1} . \lambda$ is obviously a homotopy equivalence, and by (1.6), $\tilde{\eta}_{k}^{\alpha_{k}}=$ id. By Lemma 2.2, there exists a finite union of closed intervals $U_{k}=U \subseteq[0,2 \pi)$ with total length $\left|U_{k}\right| \geq 1$, such that for any $u \in U_{k}, \exists t_{k}(u)=$ $t(u) \in \tilde{\lambda}_{k}^{-1}(u)$, such that $(2.2)$ holds. $t_{k}(u) \in \tilde{\lambda}_{k}^{-1}(u)$ means that

$$
\tilde{\Lambda} \circ \tilde{h}^{\prime}\left(q_{k}, t_{k}(u)\right)=u,
$$

and (2.2) implies

$$
\left|\tilde{\Lambda} \circ h^{\prime} \circ \tilde{f}_{n, r}\left(q_{k}, t_{k}(u)\right)-\tilde{\Lambda} \circ h^{\prime}\left(q_{k}, t_{k}(u)\right)\right| \geq 1 / \alpha_{k} .
$$

Fix some Riemannian metric on $N^{n+1}$, so $h^{\prime}\left(\stackrel{\circ}{D}^{n} \times S^{1}\right)$ is a Riemannian submanifold. The pullback by the covering map of this metric gives a Riemannian metric

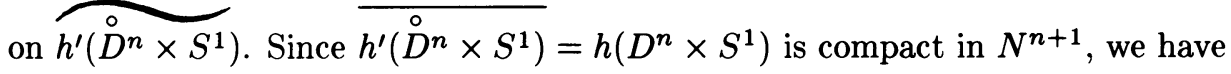

$$
\operatorname{Volume}\left(h^{\prime}\left(\stackrel{\circ}{D}^{n} \times S^{1}\right)\right)<\infty \text {. }
$$

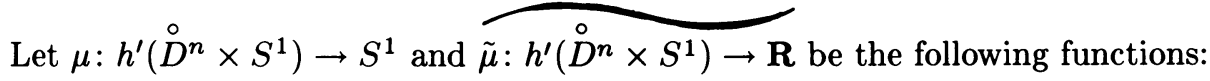

$$
\mu(p)=\Lambda \circ h^{\prime} \circ f_{n, r} \circ h^{\prime-1}(p)-\Lambda(p),
$$


and

$$
\tilde{\mu}(p)=\tilde{\Lambda} \circ \tilde{h}^{\prime} \circ \tilde{f}_{n, r} \circ \tilde{h}^{\prime-1}(p)-\tilde{\Lambda}(p) .
$$

Then $\tilde{\mu}$ is a lift of $\mu$. By (2.3),

$$
\tilde{\mu}=0 \quad \text { near } \tilde{h}^{\prime}\left(\partial Q_{k} \times \mathbf{R}^{1}\right) \quad \forall k \geq 1 .
$$

Since $\Lambda$ is $C^{\infty}$ and $h^{\prime} \circ f_{n, r} \circ h^{\prime-1}$ was supposed to be $C^{S}$ in $h^{\prime}\left(\stackrel{\circ}{D}^{n} \times S^{1}\right) \ni$ $h^{\prime}\left(\stackrel{\circ}{D}^{n}(1 / 2) \times S^{1}\right)$ we deduce by $(2.7)$ that

$$
\|\tilde{\mu}\|_{C^{s}\left(\tilde{h}^{\prime}\left(D^{n}(1 / 2) \times \mathbf{R}^{1}\right)\right)}=\|\mu\|_{C^{s}\left(h^{\prime}\left(D^{n}(1 / 2) \times S^{1}\right)\right)} \leq K_{1},
$$

for some constant $K_{1}>0$, where $\|\cdot\|_{C^{s}(X)}$ denotes the $C^{s}$-norm.

Let $\delta>0$ and $c_{1}>0$ be such that for any point $p$ in $h^{\prime}\left(D^{n}(1 / 2) \times S^{1}\right)$ the ball $B(p, \delta)$ in $N^{n+1}$ centered at $p$ of radius $\delta$ is contained in $h^{\prime}\left(\stackrel{\circ}{D}^{n} \times S^{1}\right)$ and

$$
\operatorname{Volume}(B(p, \gamma)) \geq c_{1} \gamma^{n+1} \quad \forall \gamma \in(0, \delta] \text {. }
$$

We may also assume that $\delta$ is less than the injectivity radius at any point in $h^{\prime}\left(D^{n}(1 / 2) \times S^{1}\right)$. It follows that any ball $D(p, \gamma)$ in $\overbrace{h^{\prime}\left(\stackrel{\circ}{D}^{n} \times S^{1}\right)}$, with

$$
p \in \tilde{h}^{\prime}\left(\stackrel{\circ}{D}^{n}(1 / 2) \times S^{1}\right)
$$

and $\gamma \leq \delta$, isometrically injects into $h^{\prime}\left(\stackrel{\circ}{D}^{n} \times S^{1}\right)$ by the covering map. Since $\Lambda$ is smooth on $h^{\prime}\left(\stackrel{\circ}{D}^{n} \times S^{1}\right) \supseteq h^{\prime}\left(D^{n}(1 / 2) \times S^{1}\right)$, so there is some constant $K_{2}>0$ such that

$$
\left|\tilde{\Lambda}(p)-\tilde{\Lambda}\left(p^{\prime}\right)\right| \leq K_{2} d\left(p, p^{\prime}\right) \quad \forall p, p^{\prime} \in \tilde{h}^{\prime}\left(D^{n}(1 / 2) \times \mathbf{R}^{1}\right)
$$

where $d$ is the distance in $h^{\prime}\left(\stackrel{\circ}{D^{n}} \times S^{1}\right)$.

For any $u \in U_{k}$, we let $p_{k}(u)=\tilde{h}^{\prime}\left(q_{k}, t_{k}(u)\right) \in \tilde{h}^{\prime}\left(Q_{k} \times \mathbf{R}^{1}\right)$. Then by $(2.5)$ and (2.8),

$$
\left|\tilde{\mu}\left(p_{k}(u)\right)\right| \geq 1 / \alpha_{k} .
$$

But by (2.9), $\tilde{\mu}=0$ near $\tilde{h}^{\prime}\left(\partial Q_{k} \times \mathbf{R}^{1}\right)$, and by (2.10) and (1.3), $\tilde{\mu}$ has bounded $C^{s}$-norm in $\tilde{h}^{\prime}\left(Q_{k} \times \mathbf{R}^{1}\right) \subseteq \tilde{h}^{\prime}\left(\stackrel{\circ}{D}^{n}(1 / 2) \times \mathbf{R}^{1}\right)$. So by repeated application of the Mean Value Theorem to $\tilde{\mu}$ on geodesic segments joining $p_{k}(u)$ and points on the closed subset $\tilde{h}^{\prime}\left(\partial Q_{k} \times \mathbf{R}^{1}\right)$ nearest to $p_{k}(u)$, we may obtain

$$
\operatorname{Distance}\left(p_{k}(u), \tilde{h}^{\prime}\left(\partial Q_{k} \times \mathbf{R}^{1}\right)\right) \geq c_{2}\left(1 / \alpha_{k}\right)^{1 / s},
$$

for some constant $c_{2}>0$ independent of $k$ with $c_{2}\left(1 / \alpha_{k}\right)^{1 / s} \leq \delta \forall k \geq 1$ (recall that $\left.\alpha_{k} \rightarrow \infty\right)$. This implies that the ball $B\left(p_{k}(u), c_{2}\left(1 / \alpha_{k}\right)^{1 / s}\right)$ is contained in $\tilde{h}^{\prime}\left(Q_{k} \times \mathbf{R}^{1}\right)$. But $\left|U_{k}\right| \geq 1$ and $U_{k} \subseteq[0,2 \pi)$, so there are points $u_{i} \in U_{k}, i=$ $1,2, \ldots, j_{k}$, for some

$$
j_{k} \geq\left(2 K_{2} c_{2}\left(1 / \alpha_{k}\right)^{1 / s}\right)^{-1}-2
$$


such that $K_{2} c_{2}\left(1 / \alpha_{k}\right)^{1 / s} \leq u_{1}<\cdots<u_{j_{k}}<2 \pi-K_{2} c_{2}\left(1 / \alpha_{k}\right)^{1 / s}$, and $\left|u_{i+1}-u_{i}\right|>$ $2 K_{2} c_{2}\left(1 / \alpha_{k}\right)^{1 / s}$. Then by $(2.4),(2.12)$ and the definition of $p_{k}(u)$, for any points $p \in B\left(p_{k}\left(u_{i}\right), c_{2}\left(1 / \alpha_{k}\right)^{1 / s}\right)$ and $p^{\prime} \in B\left(p_{k}\left(u_{i+1}\right), c_{2}\left(1 / \alpha_{k}\right)^{1 / s}\right)$, we have

$$
0 \leq \tilde{\Lambda}(p)<\tilde{\Lambda}\left(p^{\prime}\right)<2 \pi \text {. }
$$

It follows that $W_{k}=\bigcup_{i=1}^{j_{k}} B\left(p_{k}\left(u_{i}\right), c_{2}\left(1 / \alpha_{k}\right)^{1 / s}\right)$ is a disjoint union of balls contained in $\tilde{\Lambda}^{-1}([0,2 \pi)) \cap \tilde{h}^{\prime}\left(Q_{k} \times \mathbf{R}^{1}\right)$, with

$$
\operatorname{Volume}\left(W_{k}\right)=\sum_{i=1}^{j_{k}} \text { Volume } B\left(p_{k}\left(u_{i}\right), c_{2}\left(\frac{1}{\alpha_{k}}\right)^{1 / s}\right) \text {. }
$$

By (2.11) and (2.14), it follows that

$$
\begin{aligned}
\operatorname{Volume}\left(W_{k}\right) & \geq j_{k} c_{1}\left(c_{2}\left(1 / \alpha_{k}\right)^{1 / s}\right)^{n+1} \\
& \geq c_{3}\left(1 / \alpha_{k}\right)^{n / s}\left(1-K_{3}\left(1 / \alpha_{k}\right)^{1 / s}\right)
\end{aligned}
$$

for some constants $c_{3}>0, K_{3}>0$ independent of $k$. As $Q_{k}$ are disjoint subsets of $\stackrel{\circ}{D}^{n}, W_{k} \subset \tilde{\Lambda}^{-1}([0,2 \pi)) \cap \tilde{h}^{\prime}\left(Q_{k} \times \mathbf{R}^{1}\right)$ are all disjoint. Let $W=\bigcup W_{k}$, then by (2.16), (1.1) and the fact that $\alpha_{k}$ converges to $\infty$, we have

$$
\operatorname{Volume}(W) \geq \sum_{k=1}^{\infty} c_{3}\left(\frac{1}{\alpha_{k}}\right)^{n / s}\left(1-K_{3}\left(\frac{1}{\alpha_{k}}\right)^{1 / s}\right)=\infty .
$$

On the other hand, since $W$ is a subset of the fundamental domain $\tilde{\Lambda}^{-1}([0,2 \pi)) \subseteq$ $h^{\prime}\left(\stackrel{\circ}{D}^{n} \times S^{1}\right)$ for $h^{\prime}\left(\stackrel{\circ}{D}^{n} \times S^{1}\right)$, we have

$$
\operatorname{Volume}(W) \leq \operatorname{Volume}\left(\tilde{\Lambda}^{-1}([0,2 \pi))\right)=\operatorname{Volume}\left(h^{\prime}\left(\stackrel{\circ}{D} \times S^{2}\right)\right),
$$

which contradicts (2.6) and (2.17).

Using Theorem 2.1, all results in [H2] on the existence of nonsmoothable foliations and the density theorem for unsmoothable diffeomorphisms extend to the case $n+1=4$. (In fact, in Harrison's statement of the density theorem [H2, Corollary 4], it is permissible to use the $C^{r}$-topology rather than the $C^{0}$-topology.)

\section{REFERENCES}

[D] A. Denjoy, Sur les courbes définies par les équations différentielles à la surface du tore, J. Math. Pures Appl. 11 (1932), 333-375.

[H1] J. Harrison, Unsmoothable diffeomorphisms, Ann. of Math. 102 (1975), 85-94.

[H2] __ Unsmoothable diffeomorphisms on higher dimensional manifolds, Proc. Amer. Math. Soc. 73 (1979), 249-255.

Department of Mathematics, University of CAlifornia, SAN Diego, La Jolla, CALIFORNIA 92093-0112 